DNA. For Xenopus, Davidson et al. (J. molec. Biol., 77, 1; 1973) have presented evidence that the types of sequence are interspersed throughout the genome and they propose that 200-400 bases of unique sequence alternate with longer unique sequences of average length 600-900 nucleotides, large enough to accommodate a single gene.

It is attractive to speculate that the short repeated sequences represent control elements which regulate the transcription of adjacent unique sequences. As already noted some of these repeated sequences are transcribed and indeed tissue specificity in the repetitive sequences of in vivo RNA is demonstrable in the earlier experiments of Shearer and McCarthy (Biochemistry, 6, 283; 1967). The possibility that there is a degree of specificity in the repetitive RNA sequences from different tissues provides an explanation of why RNA excess experiments with chromatinprimed RNAs show tissue specificity. Hence within the repetitive sequences there may be broad specificities which reflect in a general way the specific mRNA sequences of a given tissue. Obviously because of the relatedness of repetitive sequences and the present uncertainty about their function this is not a particularly definitive parameter of gene expression. Hybridisation to cDNA complementary for a known mRNA clearly offers the most exact method available.

At the Cold Spring Harbor Symposium, the Glasgow group also reported results which indicated how this new approach might be used to identify molecules concerned with the restriction of chromatin. It was found that when the structure of foetal liver chromatin is disrupted by dissociating the DNA and proteins in high salt-urea solutions and then the components reassociated by slowly dialysing away the salt, the resulting chromatin still serves as a template for globin mRNA sequences. This means that the specific way in which chromatin is assembled is an inherent property of the components themselves. Which are the molecules responsible for directing this selfassembly?

A preliminary investigation implicates molecules within the nonhistone or acidic protein fraction of chromatin. Non-histone proteins from foetal liver were isolated free of DNA and histones on hydroxy- apatite columns. This material was allowed to associate with mouse brain chromatin by the reconstitution process already described. It could be shown that globin mRNA sequences which are normally absent from brain chromatin transcripts are now detected in the RNA transcribed from brain chromatin reconstituted with liver non-histone proteins. This suggests that there is a component of liver non-histone which is responsible for controlling the expression of the globin gene.

The isolation and handling of nonhistone proteins have now reached a stage where virtually pure fractions of individual components can be obtained. Although some of these are probably chromatin-associated enzymes or structural proteins rather than control elements, it is now possible for the first time to make this distinction and, hopefully by narrowing down the field, ultimately to identify the responsible molecules.

S. G.

\section{MEDICAL RADIOLOGY \\ Benefils and Risks}

from a Correspondent

Patients undergoing medical radiology are subject to small, but not negligible, genetic and somatic hazards. If the risks and benefits could be quantified, a cost/benefit study could be undertaken and measures to reduce the detriment could be assessed realistically against the likely gain. This was the main theme of a conference organised by the Society for Radiological Protection in Birmingham on October 9.

The evaluation of the risks involves four stages. First, the radiation doses to individual organs are determined by direct measurement or otherwise; next, for each organ the mean dose to the population is assessed, taking into account the number of patients involved, their age distribution and other factors; third, the biological effect of a given organ dose is estimated; finally, a monetary value is attached to a given detriment.

The radiation doses involved are known reasonably well. By far the largest contributor is diagnostic radiology and this field was reviewed by S. B. Osborn (King's College Hospital, London). The evidence is derived mainly from the 1960 and 1966 reports of the Adrian Committee. The mean genetically significant dose (GSD) from diagnostic $\mathbf{X}$ rays to the whole population of the United Kingdom is about 15 mrad $\mathrm{yr}^{-1}$, and the annual mean bone marrow dose is about $32 \mathrm{mrad}$. In other countries the GSD ranges from 1 to 50 mrad depending on the amount of radiology undertaken and the techniques used. Osborn pointed out that very large variations occur from hospital to hospital and the United Kingdom detriment could be reduced considerably if all institutions reached the standard of the best. this could not, however, be achieved without additional expenditure.

The contribution of radiotherapy to the mean annual GSD is about 5 mrad, whereas that from radioisotope tests is currently about ten times lower. The use of unsealed radioisotopes, however,

\title{
Conditions for Restriction in E. coli
}

MANY strains of Escherichia coli recognise the DNA of other strains and of phage. Recognition is followed by hydrolysis of the invading DNA, with a strain-specific endonuclease, unless the DNA is modified (by glucosylation and methylation) or mutated-presumably at the recognition site (SB site). A DNA duplex with only one strand modified is protected from degradation. Some experiments by Hartman and Zinder, of the Rockefeller University of New York, suggested that in some FI phage crosses of DNA duplexes only one strand is mutated at an SB site. They wondered how this mutant hybrid would compare with a modified hybrid when challenged with restriction enzymes and so, together with their colleagues Vovis and Horiusha, designed experiments to tackle the problem. Next Wednesday's Nature New Biology (November 7) contains an account of this work.

DNA heteroduplexes were made in vitro by annealing the wild-type DNA of phage FI with alkali denatured RFIII/PL (that is, full length, linear, double stranded DNA enzymatically prepared from the replicating form of the virus-RFI, with restriction enzyme PL). The resulting heteroduplex, RFII, is circular, double stranded with at least one single strand nick.

The phage FI has two sites $\mathrm{SB}_{1}$ and $\mathrm{SB}_{2}$ which confer susceptibility to E. coli B restriction. By making heteroduplexes in which one or other of the DNA strands was modified or mutated at the SB sites, and testing for restriction with $E$. coli enzyme B, it was possible to confirm that both modification and mutation of either strand of a DNA duplex at the SB site protect the molecule from restriction.

The authors were not able to determine whether SB mutant hybrids can be modified in vivo or in vitro, but they are at present working on this question. 